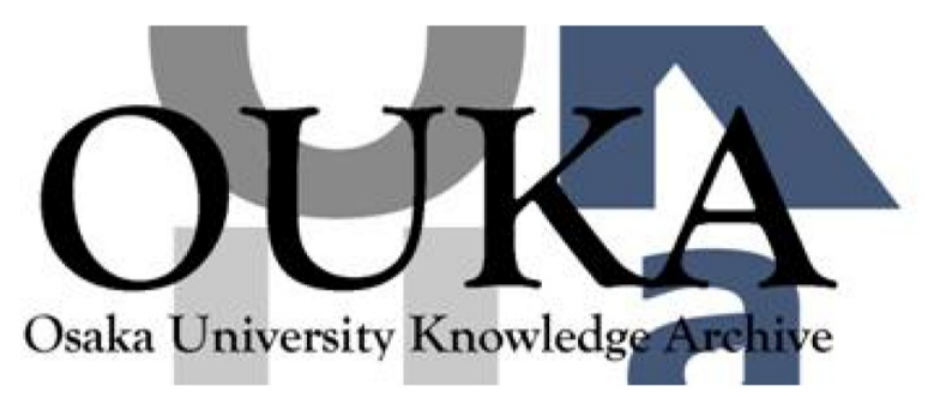

\begin{tabular}{|c|c|}
\hline Title & $\begin{array}{l}\text { Tilt orientationally disordered hexagonal } \\
\text { columnar phase of phthalocyanine discotic } \\
\text { liquid crystals }\end{array}$ \\
\hline Author (s) & $\begin{array}{l}\text { Yoneya, Makoto; Makabe, Takayoshi; Miyamoto, } \\
\text { Ayano et al. }\end{array}$ \\
\hline Citation & Physical Review E. 89(6) p.062505-p.062505 \\
\hline Issue Date & $2014-06-30$ \\
\hline oaire:version & VoR \\
\hline URL & https://hdl. handle. net/11094/75676 \\
\hline rights & $\begin{array}{l}\text { Copyright (2014) by the American Physical } \\
\text { Society }\end{array}$ \\
\hline Note & \\
\hline
\end{tabular}

Osaka University Knowledge Archive : OUKA

https://ir. Library. osaka-u. ac. jp/

Osaka University 


\title{
Tilt orientationally disordered hexagonal columnar phase of phthalocyanine discotic liquid crystals
}

\author{
Makoto Yoneya, ${ }^{*}$ Takayoshi Makabe, and Ayano Miyamoto \\ National Institute of Advanced Industrial Science and Technology, 1-1-1 Umezono, Tsukuba 305-8568, Japan \\ Yo Shimizu \\ National Institute of Advanced Industrial Science and Technology, 1-8-31 Midorioka, Ikeda, 563-8577, Japan \\ Yasuo Miyake ${ }^{\dagger}$ \\ National Institute of Advanced Industrial Science and Technology, 1-8-31 Midorioka, Ikeda, 563-8577, Japan \\ and Osaka University, 2-1 Yamada-oka, Suita, Osaka, 565-0871, Japan \\ Hiroyuki Yoshida, Akihiko Fujii, and Masanori Ozaki \\ Osaka University, 2-1 Yamada-oka, Suita, Osaka, 565-0871, Japan
}

(Received 16 January 2014; published 30 June 2014)

\begin{abstract}
The structures of the discotic liquid crystalline (LC) phase of metal-free octa-substituted phthalocyanine (Pc) derivatives were investigated using molecular dynamics (MD) simulations. Special attention was paid to the LC phase structure of the non-peripheral octa-hexyl substituted Pc-derivatives that were recently found to show very high carrier mobilities for the discotic LCs. We obtained spontaneous transition to the columnar hexagonal $\left(\mathrm{Col}_{h}\right)$ LC phase in a melting simulation from the crystal structure obtained using an x-ray diffraction study. In this simulated $\mathrm{Col}_{h}$ structure, the Pc-core normal vectors were tilted $47^{\circ}$ from the column axis in parallel within each column, but the tilting directions are disordered between columns. We also found that the inter-core distance was not as large as previously suggested $(0.4-0.5 \mathrm{~nm})$ but similar to the common value $(0.36 \mathrm{~nm})$. This may resolve the contradiction between the high carrier mobility of the non-peripheral substituted Pcs, because larger inter-core separations degrade the mobilities.
\end{abstract}

DOI: 10.1103/PhysRevE.89.062505

PACS number(s): 61.30.Cz, 61.30.Eb

\section{INTRODUCTION}

Phthalocyanines (Pcs) are well known for their rich functionalities, not only for dyes and pigments but also for electronics and optoelectronics [1]. The latter two fields have recently attracted interest on the Pcs and their derivatives [2]. Peripheral and non-peripheral alkyl substitution of Pcs (Fig. 1) enhances their solubility and also induce discotic liquid crystalline (LC) phases [3]. Solubility enables large-area, flexible, and light-weight device production by solution-processing techniques such as ink-jet printing [4]. Discotic LC materials have a promising ability to self-assemble into columnar stacks with some tendency to from their column axis oriented perpendicular (or parallel) to the substrate surfaces [5-7]. However, discotic LC materials have hardly been applied in electronics and optoelectric devices mainly because of the low carrier mobilities of $10^{-3} \mathrm{~cm}^{2} \mathrm{~V}^{-1} \mathrm{~s}^{-1}$ as a maximum value [3].

Recently, Iino et al. reported that metal-free non-peripheral octa-octyl substituted $\mathrm{Pc}\left(\mathrm{H}_{2} \mathrm{Pc}\left(\mathrm{C}_{8} \mathrm{H}_{17}\right)_{8}^{n p}\right)$ demonstrated the highest carrier mobilities of up to $0.3 \mathrm{~cm}^{2} \mathrm{~V}^{-1} \mathrm{~s}^{-1}$ in the columnar (Col) LC phase with time-of-flight (TOF) measurements [7,8]. TOF mobilities of the same order and $1.4 \mathrm{~cm}^{2} \mathrm{~V}^{-1} \mathrm{~s}^{-1}$ were reported with the octa-hexyl analog $\left(\mathrm{H}_{2} \mathrm{Pc}\left(\mathrm{C}_{6} \mathrm{H}_{13}\right)_{8}^{n p}\right)$ in its $\mathrm{Col}$ phase and crystal phase, respectively [9]. Correlated with these high mobilities, a high solar-cell efficiency of up to $3.1 \%$ was reported with a binary mixture of the $\mathrm{H}_{2} \mathrm{Pc}\left(\mathrm{C}_{6} \mathrm{H}_{13}\right)_{8}^{n p}$ with

\footnotetext{
*makoto-yoneya@aist.go.jp

${ }^{\dagger}$ Present address: JNC Corporation, Tokyo, Japan.
}

the fullerene derivative 1-(3-methoxy-carbonyl)-propyl-1-1phenyl-(6,6)C61 (PCBM) [10].

However, the origin of these high carrier mobilities is not well understood [8]. Iino et al. found that unlike most other high mobility discotics, the $\mathrm{H}_{2} \mathrm{Pc}\left(\mathrm{C}_{8} \mathrm{H}_{17}\right)_{8}^{n p}$ system shows no clear evidence of the high mobility in X-ray diffraction (XRD) studies [8]. The Norwich group suggested that the inter-core distance normal to the cores would be larger (0.44 [11], $0.4-0.5 \mathrm{~nm}[12])$ than common values (ca. $0.35 \mathrm{~nm}$ ) and there is no long-range translational stacking order of the molecules based on the XRD data (from this, they denoted the structure as "discotic disordered hexagonal" after Destrade et al. [13]). However, these larger inter-core separation and stacking disorders contradict the high carrier mobilities, because these both are known to degrade the mobilities. Iino et al. suggested that the unusual properties of this discogen must ultimately relate to the unusual molecular structure of the discogen [8]. Thus, clarification of the discotic LC phase structure of these non-peripheral substituted Pcs is necessary. Molecular simulation is one possible tool to visualize the molecular-level LC phase structure $[14,15]$ and to help understand its link to the carrier transport [16-23].

In this study, we perform molecular dynamics (MD) simulations to study the discotic LC phase structure of the $\mathrm{H}_{2} \mathrm{Pc}\left(\mathrm{C}_{6} \mathrm{H}_{13}\right)_{8}^{n p}$ and the related system. We focus here on the local LC phase structure, i.e., the inter-core distance and its stacking order. Besides these local aspects, a mesoscopicscale (ca. $100 \mathrm{~nm}$ ) defect structure is also relevant to the high TOF mobilities, because the TOF measurements are known to be sensitive to the structural defects especially in one-dimensional carrier transport systems. However, no 


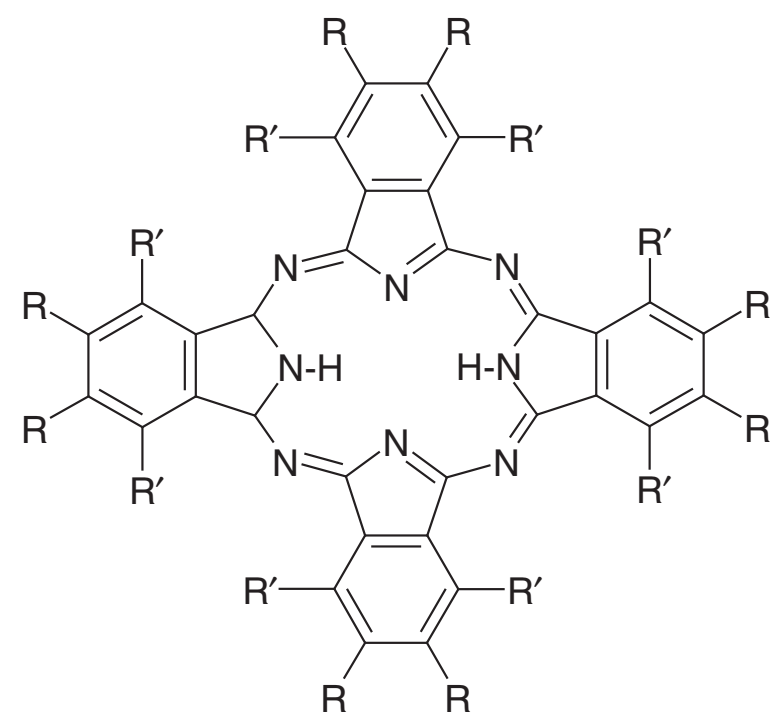

FIG. 1. Peripheral $\left(R=\mathrm{C}_{n} \mathrm{H}_{2 n+1}, R^{\prime}=\mathrm{H}\right)$ and non-peripheral ( $R=\mathrm{H}, R^{\prime}=\mathrm{C}_{n} \mathrm{H}_{2 n+1}$ ) octa-alkyl substituted phthalocyanine.

experimental information on the mesoscopic-scale structure of the system is available to best of our knowledge. We then think the contradiction between the local structure characteristics (from the XRD studies) and the high carrier mobilities described in the above would be a better target as the first step.

\section{MODEL AND METHODS}

We applied the flexible detailed-atom model with the exception that all of the bond-stretching degrees of freedom were constrained to the equilibrium bond lengths. For the inter- and intramolecular interactions, a general AMBER force field [24] was used in combination with the aliphatic $\mathrm{CH}_{2}$ and $\mathrm{CH}_{3}$ united atom parameters from a reoptimized united atom force field [25]. The restrained electrostatic potential (RESP) charges [26] were obtained using ab initio molecular orbital calculations with the B3LYP/6-31G(d) level and the GAUSSIAN03 program [27] and were used for the atomic charges.

MD simulations were conducted using the program package GROMACS (version 4.0.7) [28]. The GROMACS all-atom molecular topology file for $\mathrm{H}_{2} \mathrm{Pc}\left(\mathrm{C}_{6} \mathrm{H}_{13}\right)_{8}^{n p}$ was created using the program ACPYPE [29] as the interface for the automatic atom type and bond type perception program ANTECHAMBER [30], and the output topology file was then modified for the aliphatic $\mathrm{CH}_{2}$ and $\mathrm{CH}_{3}$ united atoms. The topology file with the atomic charge assignments that aided in reproducing the simulation in this study are presented in the Supplemental Material [31].

The $\mathrm{H}_{2} \mathrm{Pc}\left(\mathrm{C}_{6} \mathrm{H}_{13}\right)_{8}^{n p}$ molecular model above was checked by simulations starting from the crystal structures obtained by the XRD study at room temperature (293 K) [32]. The actual initial structures were made by stacking the crystal unit cells with six, six, and three times for the $a$-, $b$-, and $c$-axis directions, respectively. The systems had $108 \mathrm{H}_{2} \mathrm{Pc}\left(\mathrm{C}_{6} \mathrm{H}_{13}\right)_{8}^{n p}$ molecular models, which were placed in a triclinic $\mathrm{MD}$ cell with periodic boundary conditions.
Trajectories were produced using GROMACS with leapfrog time integration and the LINCS bond constraint [33]. The time integration step was set to 4 fs due to the stability of the LINCS algorithm and the hydrogen mass repartitioning (to be heavier while keeping total molecular mass) [34]. A cutoff distance of $0.9 \mathrm{~nm}$ was applied to the van der Waals interactions. The value $(0.9 \mathrm{~nm})$ is the same as in the developments and evaluations of the force-field parameters applied in this study [25]. The longrange Coulombic interactions were treated using a particlemesh Ewald (PME) summation with a real-space cutoff of $0.9 \mathrm{~nm}$ and the default settings (Fourier spacing $=0.12 \mathrm{~nm}$ and PME order $=4$ ).

After running the steepest descent energy minimization and initial heat-up MD runs, equilibration MD runs of lengths up to $0.2 \mathrm{~ns}$ were performed at a constant temperature of $293 \mathrm{~K}$ that was maintained using a Nose-Hoover thermostat $[35,36]$ with a coupling time constant of $0.1 \mathrm{ps}$. After these initial runs, constant pressure runs of lengths up to $0.5 \mathrm{~ns}$ were performed at normal pressure $(1 \mathrm{~atm})$. All six degrees of freedom of the MD cells were considered and allowed by applying a Parrinello-Rahman barostat [37]. Cell parameters based on the MD cell shape were averaged over the last $0.1 \mathrm{~ns}$ of the simulations (hereinafter, for average runs, the same time-span is used unless otherwise specified) and were compared with the initial cell parameters obtained from the XRD study. Simulated cell parameters were obtained with a maximum deviation of $7.7 \%$ from the values reported in the XRD study [32] (see Table $\mathrm{S} 1$ in the Supplemental Material [31]). Although the error is larger than that for the all-atom model $(5.4 \%)$, it is acceptable for the current purpose. We thus utilized the united-atom model for the rest of the studies.

\section{RESULTS AND DISCUSSION}

\section{A. Melt from crystal to LC}

We performed a melting simulation from the crystal structure at room temperature to the LC temperature. The initial structure was made by stacking the simulated crystalline structure two times in the $c$-axis directions (a total of 216 molecules). From this structure, we conducted MD runs for $30 \mathrm{~ns}$ at $438 \mathrm{~K}$ (the middle of the LC temperature range, $434 \mathrm{~K}-443 \mathrm{~K}$ ) and normal pressure. Time variations of the cell parameters based on the MD cell shape are shown in Fig. 2. As shown in this figure, the MD cell shape largely changed in the initial part of the simulation. For this part of the simulation, a Berendsen barostat [38] instead of the Parrinello-Rahman barostat was used. The average system density was decreased from $1.14 \mathrm{~g} \mathrm{~cm}^{-3}$ to $0.86 \mathrm{~g} \mathrm{~cm}^{-3}$ in this melting simulation.

To be convinced that the simulated state is not crystal but LC, we checked the molecular diffusion with a mean squared displacements (MSD) plot for the last part of the MD run. From the slope of the MSD plot (for $t=28.5-29.5 \mathrm{~ns}$ ), the diffusion constants were evaluated as $2.1 \times 10^{-10} \mathrm{~m}^{2} \mathrm{~s}$ and $0.33 \times 10^{-11} \mathrm{~m}^{2} \mathrm{~s}$ for the parallel and perpendicular to the column axis directions, respectively. The reported values for the other columnar LC systems [39] were on the order of $10^{-11} \mathrm{~m}^{2} \mathrm{~s}$ for both directions above. We guess the lager value in the column axis direction than the experimental order may be related the limited system size in this direction. Since, 


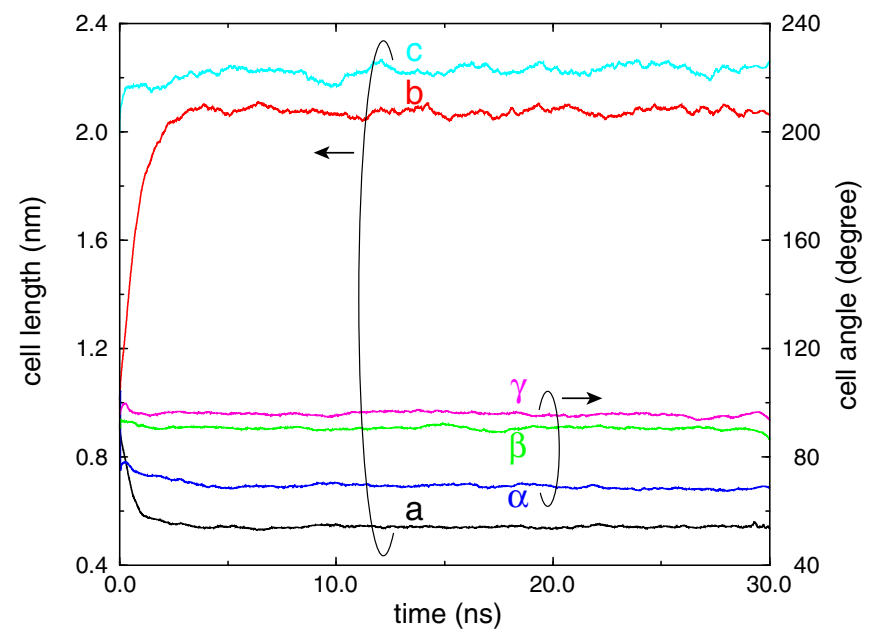

FIG. 2. (Color online) Time variations of the cell parameters in the melting simulation.

when we double the system size in the column direction, the diffusion constants were decreased to $9.5 \times 10^{-11} \mathrm{~m}^{2} \mathrm{~s}$ and $0.2 \times 10^{-11} \mathrm{~m}^{2} \mathrm{~s}$ for the parallel and perpendicular to the column axis directions, respectively. This diffusion behavior shows that the simulated state was not a crystal but a columnar LC state.

The final snapshot after a $30 \mathrm{~ns}$ MD run is shown in Fig. 3 which is viewed along the crystal $a$-axis direction and perpendicular to that. In this figure, Pc stacking columns were spontaneously developed along the crystal $a$ axis. Randomly selected single column structures are compared in Fig. 4 between the initial crystalline state and the final state in Fig. 3. The average tilt of the Pc-core normal vectors (calculated with vector product of the two connecting vectors between facing two nitrogens within the Pc-core) from the crystal $a$ axis was changed from $64^{\circ}$ to $47^{\circ}$. Usually, the tilted core structure makes rectangular column arrangements within a 2D plane perpendicular to the column axis. However, the LC phase of the $\mathrm{H}_{2} \mathrm{Pc}\left(\mathrm{C}_{6} \mathrm{H}_{13}\right)_{8}^{n p}$ was reported as a hexagonal columnar phase $[9,12,32]$. To check the column arrangement of the simulated LC structure, we calculated their structure function $S(\boldsymbol{q})$. It was calculated from

$$
S(\boldsymbol{q})=\frac{1}{N} \sum_{\alpha} e^{-i \boldsymbol{q} \cdot \boldsymbol{x}_{\alpha}},
$$

where $\boldsymbol{q}, \boldsymbol{x}_{\alpha}$, and $N$ are the scattering wave vector, the $\alpha$ th atom coordinate, and the total number of atoms, respectively [40]. The 2D plot of $|S(\boldsymbol{q})|^{2}$ for the LC structure is shown in Fig. 5. We plotted $|S(\boldsymbol{q})|^{2}$ in the $2 \mathrm{D}$ plane $\boldsymbol{q}_{x y}=\left(q_{y}, q_{z}\right)$, i.e., the vector with modulus $4 \pi \lambda^{-1} \sin \theta$, where $\lambda$ and $\theta$ are the wavelength of the incident wave vector $(0.15418 \mathrm{~nm}$ was used) and half the diffraction angle [41]. The incident wave vector direction was parallel to the column axis and the plot was averaged over the last $0.1 \mathrm{~ns}$ and shown for $2 \theta=2^{\circ}-20^{\circ}$. The 2-D structural function in Fig. 5 shows six small angle reflections in the sixfold symmetry positions, which indicates that the column arrangement of the simulated LC structure (Fig. 3) is hexagonal. From the $2 \theta$ value of these reflections, the simulated hexagonal column separation was calculated to
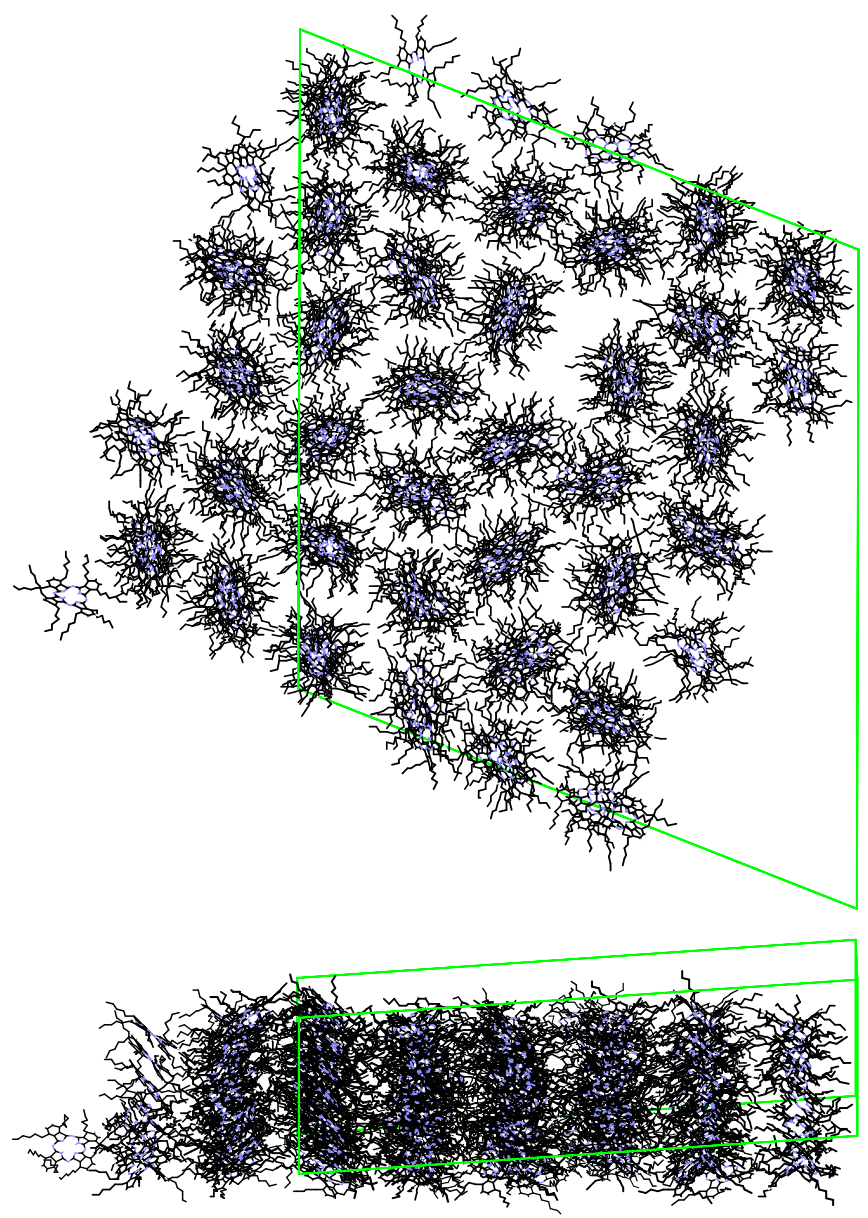

FIG. 3. (Color online) Snapshot after a $30 \mathrm{~ns}$ MD run at LC temperature $(438 \mathrm{~K})$. Upper: view along the crystal $a$ axis. Lower: view perpendicular to the crystal $a$ axis.

be $2.0 \mathrm{~nm}$. This value corresponds well to the value of $2.1 \mathrm{~nm}$ from the XRD measurements in the $\mathrm{Col}_{h}$ phase (438 K) [42]. We note that the simulated structural factors (e.g., the column separation and the Pc-core tilt) were almost not changed in the independent melting simulation with doubling the system size (in the column direction) and simulation length as shown in the Table S2 of the Supplemental Material [31].

The results in Fig. 5 imply that the simulated LC structure would be the $\mathrm{Col}_{h}$ phase. In a typical $\mathrm{Col}_{h}$ phase, the discotic cores are perpendicularly stacked against the column axis, but the Pc-cores are notably tilted from the column axis as in Fig. 4 similar to a rectangular columnar phase. Figure 6 shows that the configuration of the Pc-core normal vectors corresponds to Fig. 3 in comparison with that in the crystal phase. As shown in this figure, the Pc-core normal vectors were tilted from the column axis in parallel within each column as in the crystalline phase, but the tilting directions look intercolumnar disordered contrast to the uniaxially oriented crystal phase. Figure 7 shows the orientational correlation function $\left\langle P_{2}\left(r_{i j}\right)\right\rangle=(1 / 2)\left\langle 3\left(\boldsymbol{e}_{i} \cdot \boldsymbol{e}_{j}\right)^{2}-1\right\rangle$ Here $\boldsymbol{e}_{i}$ stands for the Pc-core normal unit vector. Solid and dashed lines correspond to the intracolumnar and intercolumnar correlation, respectively, and these were calculated and averaged over the last $0.1 \mathrm{~ns}$ of the $60 \mathrm{~ns}$ simulation with the doubly 

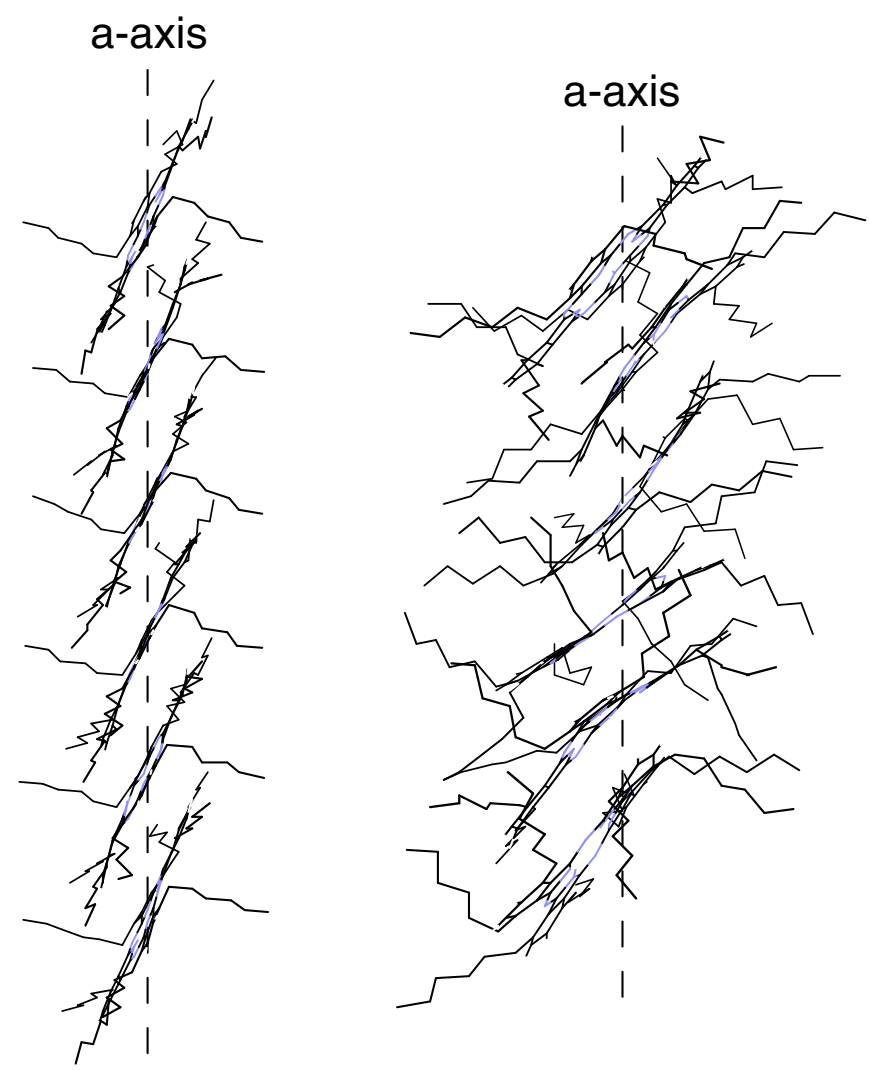

FIG. 4. (Color online) Comparison of the single column structure along the crystal $a$ axis (dashed line). Left: initial crystalline state. Right: final state in Fig. 3.

enlarged system which is described above. As in this figure, the intercolumnar tilt correlation was much shorter-ranged compare to the intracolumnar one (we note the periodicity of the intracolumnar correlation modulation roughly corresponds

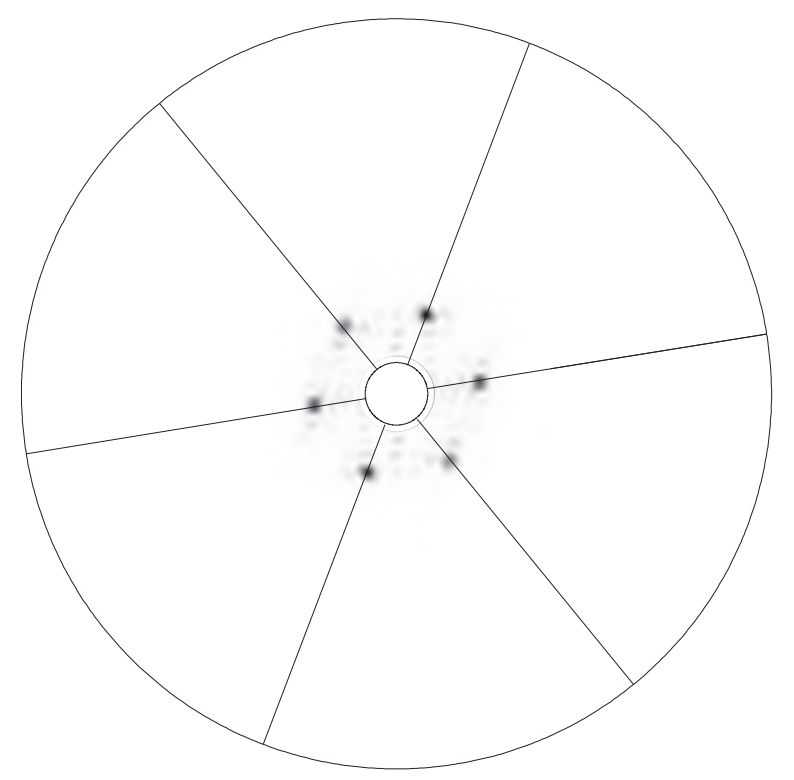

FIG. 5. Averaged 2-D structure function with the incident wave vector parallel to the column-axis.

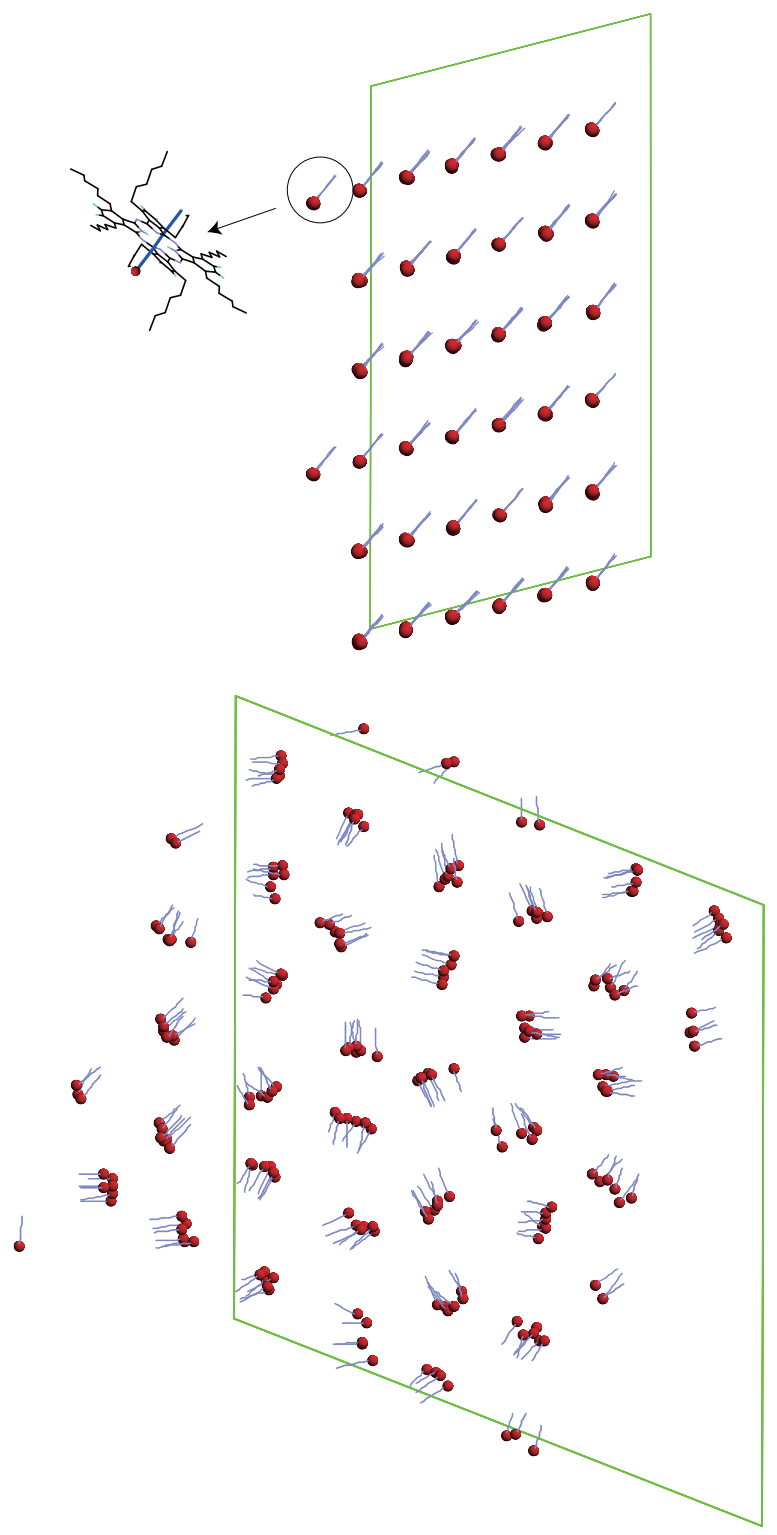

FIG. 6. (Color online) The Pc-core normal vector configurations. Upper: for the initial crystalline structure. Lower: for the snapshot in Fig. 3.

to the stacking period, i.e., $0.54 \mathrm{~nm}$ in Table S2). It clearly differs from the "pseudohexagonal" [43] structure (Fig. 6 in Ref. [44]) which has (herringbone) intercolumnar tilt correlations. These simulated characteristics correspond well to the Norwich group's disordered hexagonal columnar $\left(\mathrm{Col}_{h d}\right)$ structure model (Fig. 5 in Ref. [12]), in which the correlations of the tilting orientations are short and the lattice becomes hexagonal.

The $\mathrm{Col}_{h}$ phase generally exhibits a wide-angle XRD peak of approximately $0.35 \mathrm{~nm}$ that corresponds to the intracolumnar stacking of discotic cores [3], but we did not find this XRD peak in the current system [42]. The Norwich group already noted that the lack of this stacking peak is a remarkable characteristic of the non-peripheral alkyl substituted Pcs in general [12]. They suggested that the steric interference between the (non-peripherally substituted) alkyl 


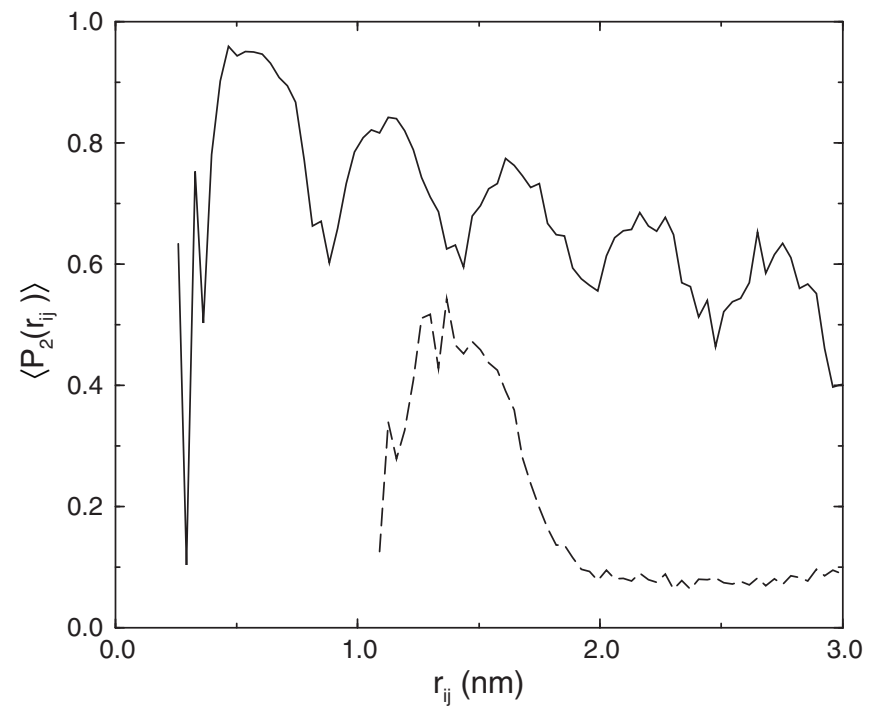

FIG. 7. Intracolumnar (solid line) and intercolumnar (dashed line) orientational correlation functions of the Pc-core normal vectors.

chains forces the disk cores further apart. This makes the peak (they considered that it corresponds to the inter-core distance normal to the cores, not the distance separating the cores along the column axis) larger than common values (e.g., $0.35 \mathrm{~nm}$ ) and coincides with the diffuse alkyl chain packing peak of approximately $0.4-0.5 \mathrm{~nm}$ [12]. In our simulation, the separation between the cores along the columnar axis (i.e., the intracolumnar stacking period) can be evaluated from the simulated cell length in the column axis directions; it was found to be $0.535 \mathrm{~nm}$ as the averaged value. If we use the average Pc-core tilt angle $\left(47^{\circ}\right)$ and the stacking period $(0.535 \mathrm{~nm})$ above, the inter-core distance normal to the cores would be $0.535 \times \cos \left(47^{\circ}\right) \sim 0.36 \mathrm{~nm}$. This suggests that the inter-core distance was not as depart from the common value (ca. $0.35 \mathrm{~nm})$ as the Norwich group suggested $(0.4-0.5 \mathrm{~nm})$.

\section{B. Tilting from a non-tilted initial structure}

In the previous section, the simulated melt from the crystal structure spontaneously transformed to the hexagonal columnar LC phase with tilted Pc-core columns. In the following, we checked this Pc-core tilting from the non-tilted (parallel stacked) initial structure. In this initial structure (see Figure S3 in the Supplemental Material [31]), the intercolumnar distance was set to the XRD value of $2.1 \mathrm{~nm}$ [42], and the intracolumnar stacking period was set to the typical value of $0.35 \mathrm{~nm}$ because this value was uncertain from the XRD data as explained in the previous section. After running the steepest descent energy minimization runs, MD runs of lengths up to $30 \mathrm{~ns}$ were performed at the LC temperature $(438 \mathrm{~K})$ and normal pressure. The snapshot after 30 ns (shown in Figure S3 in the Supplemental Material [31]) shows the spontaneous tilting of the Pc-cores like as in Fig. 3 of the previous section. The averaged Pc-core normal tilt angle, intracolumnar stacking period, and intercolumnar distance were $45.5^{\circ}, 0.518 \mathrm{~nm}$, and $2.0 \mathrm{~nm}$, respectively. The resultant inter-core distance normal to the cores was $0.36 \mathrm{~nm}$, i.e., the same as Fig. 3. Those structural values of the final structure from the non-tilted initial

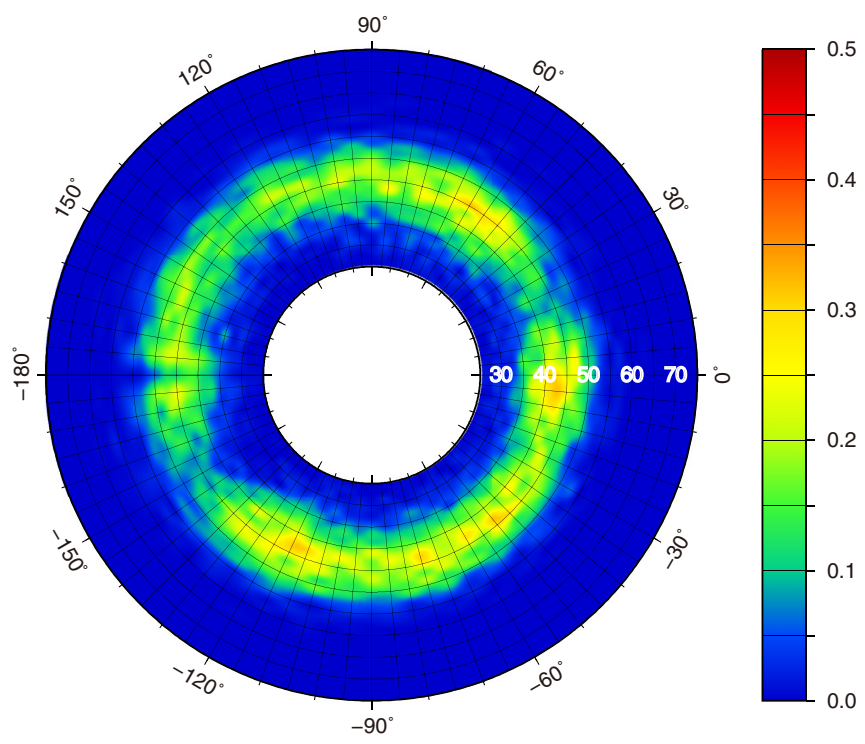

FIG. 8. (Color online) Averaged orientational distribution function of the Pc-core normal directions.

structure well correspond to those from the crystalline initial structure in the previous section. Convergence to basically the same structure from the two entirely different initial structures implies the final tilted structure can be regarded to the thermodynamically equilibrium structure.

We note again that the simulated structural factors were almost not changed in the independent spontaneous tilting simulation with a four-times larger system (in the 2D plane perpendicular to the column axis) as shown in Table S3 of the Supplemental Material [31]. Figure 8 shows that the averaged orientational distributions of the Pc-core normal directions correspond to this four-times enlarged system. The azimuthal orientations of the Pc-core normal vectors look almost uniformly distributed over a whole range of directions.

The results above indicate again that the Pc-cores are tilted in each hexagonally arranged column as in the Norwich group's $\mathrm{Col}_{h d}$ structure model [12]. Then, is this tilting specially caused by non-peripheral substitution of the terminal chains? Weber et al. proposed the same tilt disordered hexagonal structure for "peripheral" octa-alkyl substituted Pcs [43]. They deduced a Pc-core tilting angle from the stacking period $(0.49 \mathrm{~nm})$ and a close-packed distance of Pc-cores $(0.34 \mathrm{~nm})$ with $\cos ^{-1}(0.34 / 0.49)=46^{\circ}$. Here, the intracolumnar stacking period $0.49 \mathrm{~nm}$ was evaluated from the slope of the squared intercolumnar distance vs. the number of methylene groups plot. They think hexagonal packing of these tilted Pc-core columns is achieved with complete rotational disorder about the column axis [43]. For this "peripheral" octa-alkyl substituted Pcs, there was discussion on the other non-tilted column structure model [44]. Studies of oriented samples (e.g., [45]) were suggested to determine the adequate model [44] but, to the best of our knowledge, a conclusive result is not yet available.

We then performed a simulation of the "peripheral" octahexyl substituted $\mathrm{Pc}$ derivatives, $\mathrm{H}_{2} \mathrm{Pc}\left(\mathrm{C}_{6} \mathrm{H}_{13}\right)_{8}^{p}$, which shows $\mathrm{Col}_{h}$ phase in the temperature range of $523 \mathrm{~K}-636 \mathrm{~K}$ [44]. In this case, the initial intracolumnar distance was set to the 
XRD value of $2.25 \mathrm{~nm}$ [44], and the same intracolumnar stacking period of $0.35 \mathrm{~nm}$ as the previous case was used for the initial structure. A snapshot after $30 \mathrm{~ns}$ of the MD run (see Figure S5 in the Supplemental Material [31]) showed the similar spontaneous Pc-core tilting as with the $\mathrm{H}_{2} \mathrm{Pc}\left(\mathrm{C}_{6} \mathrm{H}_{13}\right)_{8}^{n p}$ system, and it supports the Weber's structure model for the peripheral octa-alkyl substituted Pcs. The averaged Pccore normal tilt angle, intracolumnar stacking period, and intercolumnar distance were $32^{\circ}, 0.41 \mathrm{~nm}$, and $2.3 \mathrm{~nm}$, respectively. The resultant inter-core distance normal to the cores was $0.35 \mathrm{~nm}$.

The result above indicates that the Pc-core tilting is not exclusive to the non-peripheral substitution but also occurs for peripheral substitution of the alkyl side chains of Pcs. Such finite core tilting in the $\mathrm{Col}_{h}$ phase was also reported based on the high-resolution synchrotron XRD study even in non-Pc compounds $[46,47]$. In this study, the tilt remains a finite value (around $20^{\circ}$ ) in the $\mathrm{Col}_{h}$ phase with the transition from the $\mathrm{Col}_{r}$ phase. The authors proposed that the $\mathrm{Col}_{r}-\mathrm{Col}_{h}$ structural phase change (in their samples) is characterized primarily by the onset of the intercolumnar disordering of the molecular tilt orientation about the column axis without large alteration of the tilt angles. Actually, in the $\mathrm{H}_{2} \mathrm{Pc}\left(\mathrm{C}_{8} \mathrm{H}_{17}\right)_{8}^{n p}$ system, which is known to have both $\mathrm{Col}_{h}$ and $\mathrm{Col}_{r}$ phases, there is almost no smallest angle XRD peak position shift (Fig. 4 of Ref. [11]) and no discontinuities in charge carrier mobilities across their $\mathrm{Col}_{r}-\mathrm{Col}_{h}$ transitions [7]. These evidences imply that there would be almost no Pc-core tilt angle change across the $\mathrm{Col}_{r}$ $\mathrm{Col}_{h}$ transitions.

So far, all the simulated octa-hexyl substituted Pc derivatives showed the tilted Pc-core structures. In the following, we checked whether our models were capable of showing nontilted Pc-core structures for the Pc derivatives that are experimentally known to have the $\mathrm{Col}_{h}$ phase with non-tilted Pc-core columns. We specifically chose the octa-pentyloxycarbonylsubstituted $\mathrm{Pc}$ derivatives, $\mathrm{H}_{2} \mathrm{Pc}\left(\mathrm{COOC}_{5} \mathrm{H}_{11}\right)_{8}^{p}$, which have a $\mathrm{Col}_{h}$ phase over a wide temperature range (at least $213 \mathrm{~K}-573 \mathrm{~K}$ ) [48]. In the corresponding initial structure, the intracolumnar distance and the intracolumnar stacking period were set to $2.502 \mathrm{~nm}$ and $0.35 \mathrm{~nm}$, based on the XRD results [48]. As in the snapshot after $30 \mathrm{~ns}$ of the MD run at 438 K (shown Figure S6 in the Supplemental Material [31]), this octa-pentyloxycarbonyl-substituted Pc derivative showed almost parallel stacking with a far less tilted Pc-core in contrast to the octa-hexyl substituted analogs.

Figure 9 shows the time variations of the averaged tilt angles of the Pc-core normal vectors for the all the simulations so far explained. We note that all the plots except the black one (melting simulation from the crystal) actually started from zero tilt initial structures, but those look to start from the finite tilt angles in this figure. This is because of rapid spontaneous tilting during the initial start-up runs. The averaged Pc-core normal tilt angle of the octa-pentyloxycarbonyl-substituted derivative was $10.1^{\circ}$; in fact, far smaller than the other octa-hexyl substituted Pc derivatives. We hypothesize that the carbonyl group in the terminal chains promotes parallel stacking of the Pc-cores. The ether group may also have the same effect, because octa-alkoxy-substituted Pcs are also known to show parallel Pc-core stacking [43]. Without these polar groups within side chains (e.g., alkyl groups), the

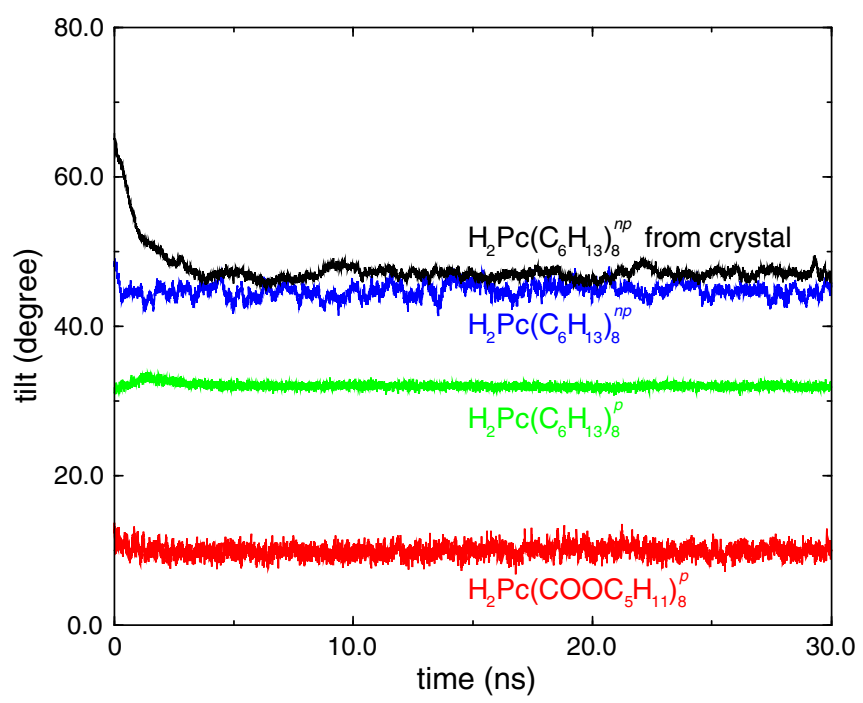

FIG. 9. (Color online) Time variations of the Pc-core normal tilts from the column axis. Black and blue lines: the $\mathrm{H}_{2} \mathrm{Pc}\left(\mathrm{C}_{6} \mathrm{H}_{13}\right)_{8}^{n p}$ system from the crystal and the non-tilted initial structure, respectively (note the convergence of them). Green line: the $\mathrm{H}_{2} \mathrm{Pc}\left(\mathrm{C}_{6} \mathrm{H}_{13}\right)_{8}^{p}$ system. Red line: the $\mathrm{H}_{2} \mathrm{Pc}\left(\mathrm{COOC}_{5} \mathrm{H}_{11}\right)_{8}^{p}$ system.

Pc-cores themselves may naturally stack with tilts like the non-substituted Pcs [43].

The averaged intracolumnar stacking period and intercolumnar distance of the octa-pentyloxycarbonyl-substituted derivative were $0.35 \mathrm{~nm}$ and $2.5 \mathrm{~nm}$, respectively. The peak corresponding to the stacking period $(0.35 \mathrm{~nm})$ was clearly seen in the averaged 1D structural functions [obtained with circular average of the 2D structural function in Eq. (1) and average over $x y z$ three incident beams] shown in Fig. 10. In contrast, the other octa-hexyl substituted Pcs only show the broad peak approximately $0.4 \mathrm{~nm}$ in the wide-angle region.

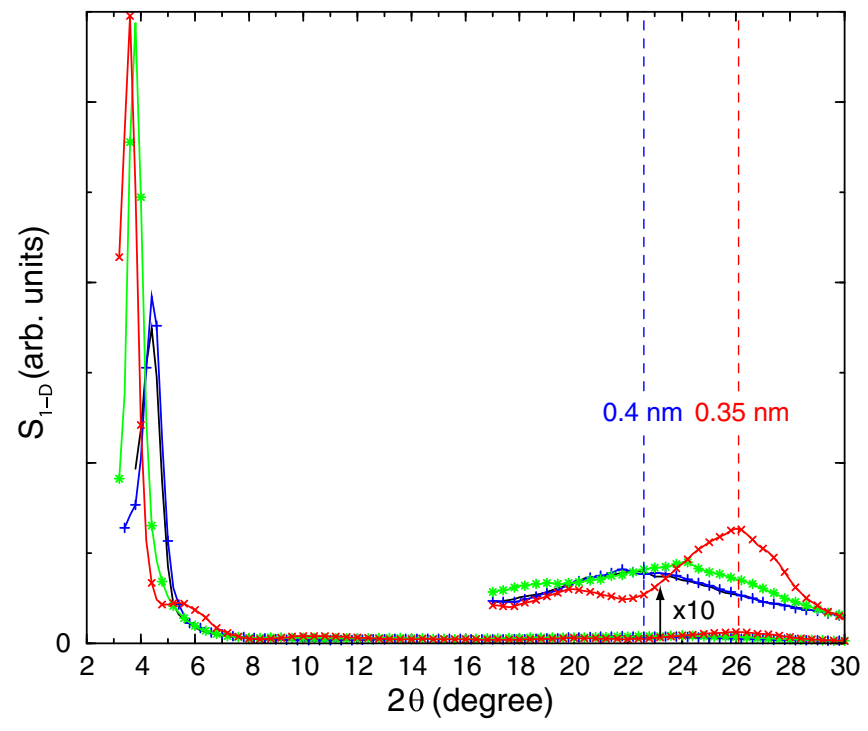

FIG. 10. (Color online) Averaged 1D structure functions. Black and blue lines (with symbol "+"): the $\mathrm{H}_{2} \mathrm{Pc}\left(\mathrm{C}_{6} \mathrm{H}_{13}\right)_{8}^{n p}$ system from the crystal and the non-tilted initial structure, respectively (note the agreement between them). Green line (with symbol “*”): the $\mathrm{H}_{2} \mathrm{Pc}\left(\mathrm{C}_{6} \mathrm{H}_{13}\right)_{8}^{p}$ system. Red line (with symbol “ $\times$ ”): the $\mathrm{H}_{2} \mathrm{Pc}\left(\mathrm{COOC}_{5} \mathrm{H}_{11}\right)_{8}^{p}$ system. 


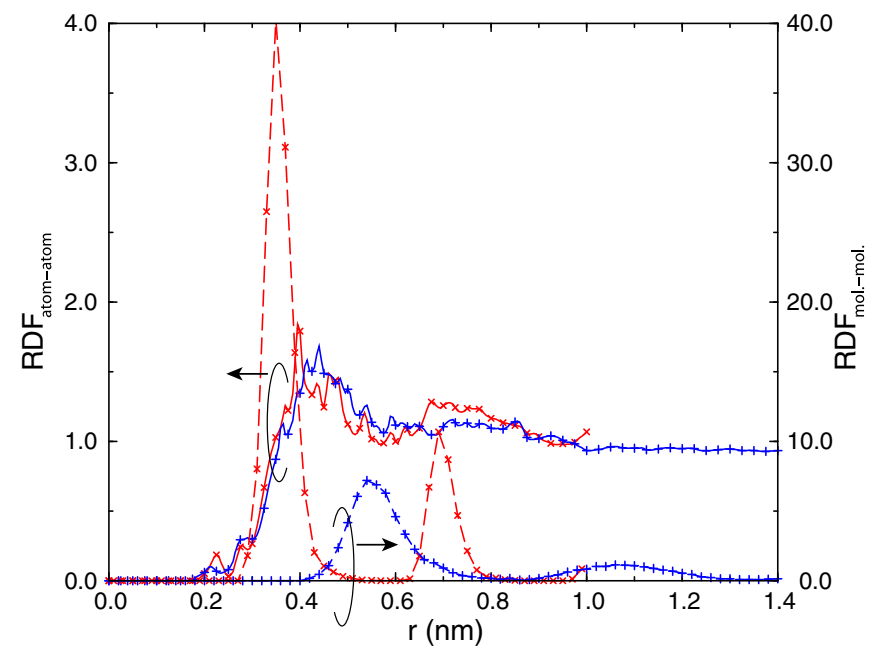

FIG. 11. (Color online) Averaged radial distribution functions. Blue lines (with symbol "+"): atom-atom (solid line) and molecularmolecular (dashed line) RDF for the $\mathrm{H}_{2} \mathrm{Pc}\left(\mathrm{C}_{6} \mathrm{H}_{13}\right)_{8}^{n p}$ system, respectively. Red lines (with symbol " $\times$ "): atom-atom (solid line) and molecular-molecular (dashed line) RDF for the $\mathrm{H}_{2} \mathrm{Pc}\left(\mathrm{COOC}_{5} \mathrm{H}_{11}\right)_{8}^{p}$ system, respectively.

Stacking peak corresponding to $0.35 \mathrm{~nm}$ for the $\mathrm{H}_{2} \mathrm{Pc}\left(\mathrm{COOC}_{5} \mathrm{H}_{11}\right)_{8}^{p}$ system above can also be identified in the molecular-molecular radial distribution functions ( $\mathrm{RDF}_{\text {mol.-mol. }}$ ) shown in Fig. 11 (red dashed line). Whereas, the $\mathrm{RDF}_{\text {mol.-mol. }}$ peak around $0.52 \mathrm{~nm}$ for the $\mathrm{H}_{2} \mathrm{Pc}\left(\mathrm{C}_{6} \mathrm{H}_{13}\right)_{8}^{n p}$ system (blue dashed line) corresponds well to the stacking period evaluated from the simulated cell length $(0.518 \mathrm{~nm})$ in the previous section. Thus, the $\mathrm{RDF}_{\text {mol.-mol. for these }}$ two systems $\left[\mathrm{H}_{2} \mathrm{Pc}\left(\mathrm{C}_{6} \mathrm{H}_{13}\right)_{8}^{n p}\right.$ and $\left.\mathrm{H}_{2} \mathrm{Pc}\left(\mathrm{COOC}_{5} \mathrm{H}_{11}\right)_{8}^{p}\right]$ are shifted in Fig. 11 because of their stacking period difference. We also evaluated the inter-core distance normal to the Pc-cores previously, and that was $0.36 \mathrm{~nm}$ and $0.35 \mathrm{~nm}$ for the $\mathrm{H}_{2} \mathrm{Pc}\left(\mathrm{C}_{6} \mathrm{H}_{13}\right)_{8}^{n p}$ and the $\mathrm{H}_{2} \mathrm{Pc}\left(\mathrm{COOC}_{5} \mathrm{H}_{11}\right)_{8}^{p}$ systems, respectively. Corresponding shoulder peaks can be found in the $\mathrm{RDF}_{\text {atom-atom }}$ in Fig. 11 (solid lines). In contrast to the $\mathrm{RDF}_{\text {mol.-mol. }}$, there is no large shift between the $\mathrm{RDF}_{\text {atom-atom }}$ 's for those two systems.

\section{CONCLUSIONS}

We obtained simulation results that showed that the Pccores were distinctly tilted from the column-axis in their columnar LC phase in both peripheral and non-peripheral octa-hexyl substituted Pcs. The tilted Pc-cores in these octahexyl substituted Pcs are arranged in hexagonal lattices, because of the intercolumnar disorder of the Pc-core tilting orientations. We think that these structure aspects could be regarded as a discotic analog to the "de Vries" smectic phase $[49,50]$ in calamitic liquid crystals. Characteristics of the aforementioned structures coincide with Weber's model and the Norwich group's model for peripheral and non-peripheral octa-alkyl substituted Pcs, respectively, and strongly support those structure models. This is the first supporting study for these structure models for the Pc systems to the best of our knowledge.

Another suggestion of the Norwich group that the intercore distance in these non-peripheral series would be larger $(0.4-0.5 \mathrm{~nm})$ than the common values (ca. $0.35 \mathrm{~nm})$ [12] may contradict with the recently reported high carrier mobilities of non-peripheral octa-alkyl substituted Pcs [7-9]. However, our simulation results showed that the inter-core distance would be similar to the common value $(0.36 \mathrm{~nm})$. This may resolve the contradiction above, but it does not explain the high carrier mobilities. A search for the origin of the high carrier mobilities is now underway.

\section{ACKNOWLEDGMENTS}

We would like to thank Dr. Fabien Nekelson of Advanced Industrial Science and Technology (now in BASF) and Dr. Michael K. Engel of DIC for their valuable discussions. This work was supported by the Japan Science and Technology Agency (JST-ALCA project).
[1] N. B. McKeown, Phthalocyanine Materials: Synthesis, Structure, and Function (Cambridge University Press, Cambridge, 1998), Vol. 6.

[2] S. Sergeyev, W. Pisula, and Y. H. Geerts, Chem. Soc. Rev. 36, 1902 (2007).

[3] S. Kumar, Chemistry of Discotic Liquid Crystals: From Monomers to Polymers (CRC Press LLC, Boca Raton, FL, 2010).

[4] K. Petritsch, J. Dittmer, E. Marseglia, R. Friend, A. Lux, G. Rozenberg, S. Moratti, and A. Holmes, Sol. Energ. Mat. Sol. C 61, 63 (2000).

[5] K. Petritsch, R. Friend, A. Lux, G. Rozenberg, S. Moratti, and A. Holmes, Synthetic Met. 102, 1776 (1999).

[6] L. Schmidt-Mende, A. Fechtenkötter, K. Müllen, E. Moons, R. Friend, and J. MacKenzie, Science 293, 1119 (2001).

[7] H. Ino, Y. Takayashiki, J.-i. Hanna, and R. J. Bushby, Jpn. J. Appl. Phys. 2 44, L1310 (2005).
[8] H. Iino, J.-i. Hanna, R. J. Bushby, B. Movaghar, B. J. Whitaker, and M. J. Cook, Appl. Phys. Lett. 87, 132102 (2005).

[9] Y. Miyake, Y. Shiraiwa, K. Okada, H. Monobe, T. Hori, N. Yamasaki, H. Yoshida, M. J. Cook, A. Fujii, M. Ozaki et al., Appl. Phys. Express 4, 021604 (2011).

[10] T. Hori, Y. Miyake, N. Yamasaki, H. Yoshida, A. Fujii, Y. Shimizu, and M. Ozaki, Appl. Phys. Express 3, 101602 (2010).

[11] A. Cherodian, A. Davies, R. Richardson, M. Cook, N. McKeown, A. Thomson, J. Feijoo, G. Ungar, and J. Harrison, Mol. Cryst. Liq. Cryst. 196, 103 (1991).

[12] A. Cammidge, M. Cook, S. Haslam, R. Richardson, and K. Harrison, Liq. Cryst. 14, 1847 (1993).

[13] C. Destrade, P. Foucher, H. Gasparoux, N. H. Tinh, A. Levelut, and J. Malthete, Mol. Cryst. Liq. Cryst. 106, 121 (1984).

[14] I. Ono and S. Kondo, Bull. Chem. Soc. Jpn. 65, 1057 (1992). 
[15] V. Marcon, T. Vehoff, J. Kirkpatrick, C. Jeong, D. Y. Yoon, K. Kremer, and D. Andrienko, J. Chem. Phys. 129, 094505 (2008).

[16] J. Tant, Y. H. Geerts, M. Lehmann, V. De Cupere, G. Zucchi, B. W. Laursen, T. Bjørnholm, V. Lemaur, V. Marcq, A. Burquel et al., J. Phys. Chem. B 109, 20315 (2005).

[17] J. Kirkpatrick, V. Marcon, J. Nelson, K. Kremer, and D. Andrienko, Phys. Rev. Lett. 98, 227402 (2007).

[18] J. Kirkpatrick, V. Marcon, K. Kremer, J. Nelson, and D. Andrienko, J. Chem. Phys. 129, 094506 (2008).

[19] Y. Olivier, L. Muccioli, V. Lemaur, Y. Geerts, C. Zannoni, and J. Cornil, J. Phys. Chem. B 113, 14102 (2009).

[20] A. Troisi, D. L. Cheung, and D. Andrienko, Phys. Rev. Lett. 102, 116602 (2009).

[21] X. Feng, V. Marcon, W. Pisula, M. R. Hansen, J. Kirkpatrick, F. Grozema, D. Andrienko, K. Kremer, and K. Müllen, Nat. Mater. 8, 421 (2009).

[22] V. Marcon, D. W. Breiby, W. Pisula, J. Dahl, J. Kirkpatrick, S. Patwardhan, F. Grozema, and D. Andrienko, J. Am. Chem. Soc. 131, 11426 (2009).

[23] F. May, V. Marcon, M. R. Hansen, F. Grozema, and D. Andrienko, J. Mater. Chem. 21, 9538 (2011).

[24] J. Wang, R. M. Wolf, J. W. Caldwell, P. A. Kollman, and D. A. Case, J. Comput. Chem. 25, 1157 (2004).

[25] L. Yang, C.-h. Tan, M.-J. Hsieh, J. Wang, Y. Duan, P. Cieplak, J. Caldwell, P. A. Kollman, and R. Luo, J. Phys. Chem. B 110, 13166 (2006).

[26] C. I. Bayly, P. Cieplak, W. Cornell, and P. A. Kollman, J. Chem. Phys. 97, 10269 (1993)

[27] M. J. Frisch, G. W. Trucks, H. B. Schlegel, G. E. Scuseria, M. A. Robb, J. R. Cheeseman, J. J. A. Montgomery, T. Vreven, K. N. Kudin, J. C. Burant et al., Gaussian03, Revision C.02 (Gaussian, Inc., Wallingford, CT, 2004).

[28] E. Lindahl, B. Hess, and D. van der Spoel, J. Mol. Model. 7, 306 (2001).

[29] A. W. S. da Silva and W. F. Vranken, BMC Res. Notes 5, 367 (2012).
[30] J. Wang, W. Wang, P. Kollman, and D. Case, J. Mol. Graphics Model. 25, 247 (2006).

[31] See Supplemental Material at http://link.aps.org/supplemental/ 10.1103/PhysRevE.89.062505 for details of the simulation.

[32] I. Chambrier, M. J. Cook, M. Helliwell, and A. K. Powell, J. Chem. Soc., Chem. Commun. 444 (1992).

[33] B. Hess, H. Bekker, H. J. C. Berendsen, and J. G. E. M. Fraaije, J. Comput. Chem. 18, 1463 (1997).

[34] K. A. Feenstra, B. Hess, and H. J. Berendsen, J. Comput. Chem. 20, 786 (1999).

[35] S. Nosé, Mol. Phys. 52, 255 (1984).

[36] W. G. Hoover, Phys. Rev. A 31, 1695 (1985).

[37] M. Parrinello and A. Rahman, J. Appl. Phys. 52, 7182 (1981).

[38] H. J. C. Berendsen, J. P. M. Postma, W. F. van Gunsteren, A. DiNola, and J. R. Haak, J. Chem. Phys. 81, 3684 (1984).

[39] R. Dong, D. Goldfarb, M. Moseley, Z. Luz, and H. Zimmermann, J. Phys. Chem. 88, 3148 (1984).

[40] P. M. Chaikin and T. C. Lubensky, Principles of Condensed Matter Physics (Cambridge University Press, Cambridge, 1995), Chap. 2.

[41] J. M. Seddon, in Handbook of Liquid Crystals, edited by D. Demus, J. Goodby, G. W. Gray, H. W. Spiess, and V. Vill (Wiley-VCH, Weinheim, 1998), Vol. 1, Chap.VIII, pp. 635-679.

[42] Y. Miyake, Ph.D. thesis, University of Osaka, 2011.

[43] P. Weber, D. Guillon, and A. Skoulios, Liq. Cryst. 9, 369 (1991).

[44] M. Engel, P. Bassoul, L. Bosio, H. Lehmanns, M. Hanacks, and J. Simon, Liq. Cryst. 15, 709 (1993).

[45] C. Grigoriadis, N. Haase, H.-J. Butt, K. Müllen, and G. Floudas, Soft Matter 7, 4680 (2011).

[46] C. R. Safinya, K. S. Liang, W. A. Varady, N. A. Clark, and G. Andersson, Phys. Rev. Lett. 53, 1172 (1984).

[47] C. Safinya, N. A. Clark, K. Liang, W. A. Varady, and L. Chiang, Mol. Cryst. Liq. Cryst. 123, 205 (1985).

[48] L. Dulog and A. Gittinger, Mol. Cryst. Liq. Cryst. 213, 31 (1992).

[49] A. de Vries, Mol. Cryst. Liq. Cryst. 41, 27 (1977).

[50] A. de Vries, J. Chem. Phys. 71, 25 (1979). 